Distribution Category:

Magnetic Fusion Energy Systems

(UC-424)

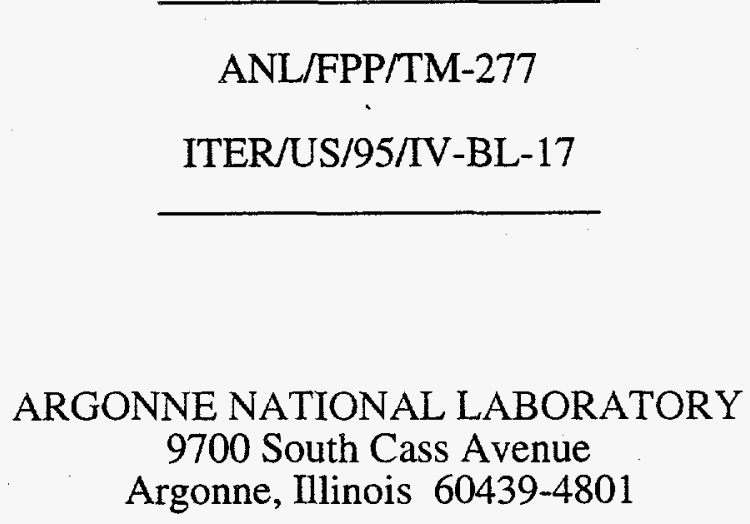

Argonne, lllinois 60439-4801

\title{
SUMMARY REPORT FOR ITER TASK - D10: \\ UPDATE AND IMPLEMENTATION OF NEUTRON TRANSPORT AND ACTIVATION CODES AND PROCESSED LIBRARIES
}

by

Hosny Attaya

Fusion Power Program/Technology Development Division

January 1995

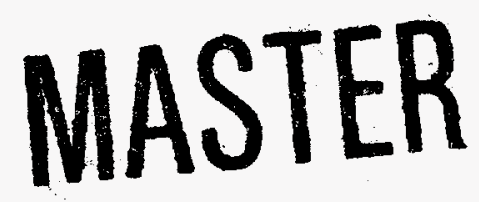

Work supported by the

Office of Fusion Energy

U.S. Department of Energy

under Contract W-31-109-Eng-38

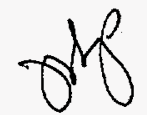




\section{DISCLAIMER}

This report was prepared as an account of work sponsored by an agency of the United States Government. Neither the United States Government nor any agency thereof, nor any of their employees, make any warranty, express or implied, or assumes any legal liability or responsibility for the accuracy, completeness, or usefulness of any information, apparatus, product, or process disclosed, or represents that its use would not infringe privately owned rights. Reference herein to any specific commercial product, process, or service by trade name, trademark, manufacturer, or otherwise does not necessarily constitute or imply its endorsement, recommendation, or favoring by the United States Government or any agency thereof. The views and opinions of authors expressed herein do not necessarily state or reflect those of the United States Government or any agency thereof. 


\section{DISCLAIMER}

Portions of this document may be illegible in electronic image products. Images are produced from the best available original document. 


\section{TABLE OF CONTENTS}

Page

\section{ABSTRACT}

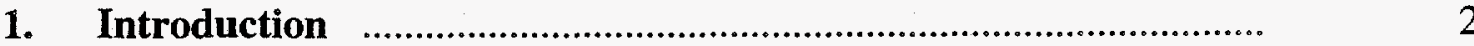

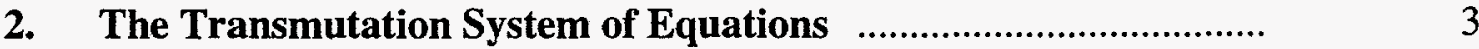

3. Methods of Solutions

3.1 Linear Chain Method .........................................................

3.2 Ordinary Differential Equation Methods ................................. 6

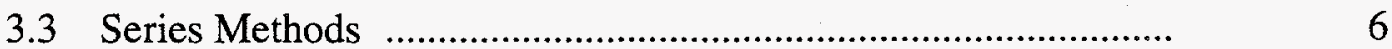

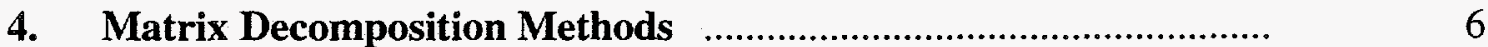

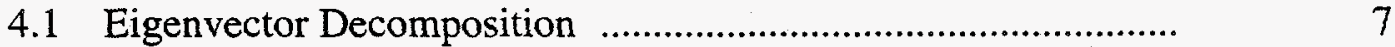

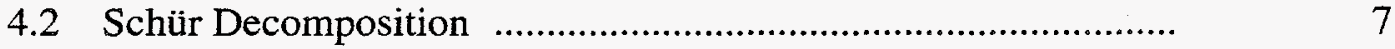

4.3 Matrix Decomposition Algorithm …................................... 8

4.4 The Padé Approximation ....................................................

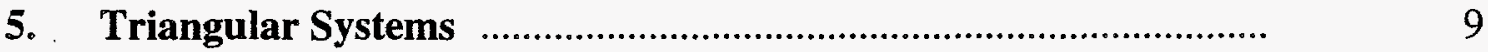

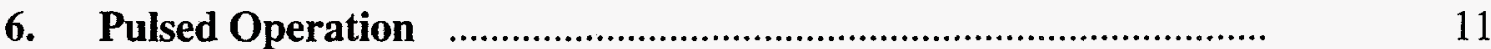

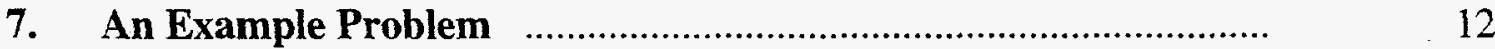

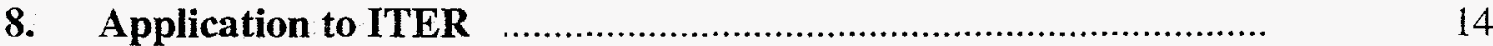

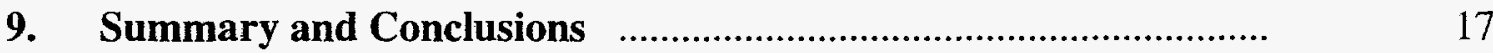

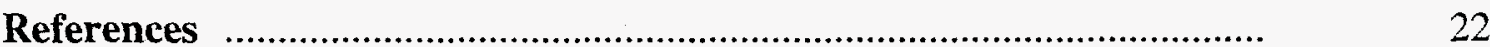




\section{LIST OF FIGURES}

Figure No.

Page

1 The transmutation matrix of the ${ }^{50} \mathrm{Cr}$.

2 The mid-plane radial build and the neutron flux of the ITER SS/water design.

3 The specific activity in the ITER inboard first wall after shutdown for the three operation scenarios.

$4 \quad$ The specific decay heat in the ITER inboard first wall after shutdown for the three operation scenarios.

5 The contact dose of the ITER inboard first wall after shutdown for the three operation scenarios.

\section{LIST OF TABLES}

Table No.

Page

1 Solution Comparison - Transmutation of ${ }^{50} \mathrm{Cr}$ after one year of continuous operation

Transmutation of ${ }^{50} \mathrm{Cr}$ after 10000 pulses 


\title{
SUMMARY REPORT FOR ITER TASK - D10: \\ UPDATE AND IMPLEMENTATION OF NEUTRON TRANSPORT AND \\ ACTIVATION CODES AND PROCESSED LIBRARIES
}

\author{
H. Attaya
}

\begin{abstract}
The primary goal of this task is to provide the capabilities in the activation code RACC, to treat pulsed operation modes. In addition, it is required that the code utilizes the same spatial mesh and geometrical models as employed in the one or multidimensional neutron transport codes used in ITER design. This would ensure the use of the same neutron flux generated by those codes to calculate the different activation parameters. It is also required to have the capabilities for generating graphical outputs for the calculated activation parameters.
\end{abstract}

All of these goals have been achieved. The major part of the work centered on developing new general and efficient mathematical methods and algorithms that can provide accurate solutions of the transmutation system of equations and can be utilized easily for the pulsed operation modes. The key for the treatment of the pulsed operation, as will be explained in detail, is to express the solution of the transmutation system of equations in terms of the transition matrix (the resolvent). The coefficients of this matrix depend only on the transmutation matrix and the time difference $\Delta t$. As a result, the solution after any number of pulses, each of which has the same $\Delta t$, can be obtained by using the same resolvent. This leads to significant computational time saving if compared to other methods that march in time blindly solving the same problem over and over again without taking advantage of the constancy of the transition matrix.

During the work we have developed and tested several algorithms based on several mathematical methods. The methods are the Schür decomposition, the eigenvector decomposition, and the Pade' approximation of the matrix exponential function. We have also developed a simple algorithm to evaluate the transition matrix for triangular transmutation system which occurs during shutdown periods.

As it turned out, the Schür decomposition method proved to be the most accurate method and among the most efficient methods. 


\section{Introduction}

A major part of the ITER Task D-10 is the upgrade of the radioactivity code RACC[5] for the conducting ITER design analysis. The other part deals with processing up-to-date activation cross-section data files and decay data for the activation calculations. The latter part has been done early in the work and is described elsewhere in this report. We focus here on upgrade and the development of the RACC code[6].

It is important to accurately determine the different safety aspects of ITER. This requires accurate radioactivity calculations for the different parts of the machine using realistic neutron flux estimates, accurate activation cross sections and decay data, as well as reasonable modeling of the ITER's pulsed operation mode.

To use accurate neutron fluxes, the code should be able to interface with any neutron transport code expected to be used for the ITER design. For this reason, the RACC code has been modified to include interfaces to the ONE/TWODANT [26]code in addition to the already existing interfaces for many transport codes.

As a result of using large activation cross section and decay data libraries[2], the computational time of activation calculations for large system as ITER, has increased considerably. Furthermore, if any of the current traditional methods used to solve the transmutation system of equations is employed to model the pulsed operation, the computational cost would be prohibited. For these reasons, it was imperative to seek different methods of solution that reduce the computational time and can be easily adopted to the treatment of the pulsed operation.

In this work, we have developed algorithms based on several mathematical methods that were chosen based on their generality, reliability, stability, accuracy, and efficiency. These methods are the matrix Schür decomposition, the eigenvector decomposition, and the Padé approximation for the matrix exponential functions. In Section 2, the characteristics of the transmutation system of equation are discussed. Section 3 presents a brief review of the currently used methods. In Section 4, the new methods are presented. A new method for solving linear chains is introduced 
in Section 5. The pulsed operation is discussed in Section 6. In Section 7, a simple problem is used to demonstrate the results of the different methods in the steadystate operation. The same problem is used to show the results of the pulsed operation algorithm which is based on the matrix decomposition methods. Finally, in Section 8 , the most recent ITER design is used to show the effect of the pulsed operation on the activation results.

\section{The Transmutation System of Equations}

The radioactivity inventory in a nuclear reactor is described by a set of simultaneous first-order linear differential equations. These equations can be written in a matrix notation as:

$$
\dot{y}=A y=(\phi C+D) y
$$

where

$y=$ the concentration vector of all the nuclides involved,

$\phi=$ the position-averaged and energy-averaged neutron flux,

$C=$ the microscopic cross-section matrix whose off-diagonal elements $c_{i j}=\sigma_{i j}$ are the spectrum-averaged microscopic cross-sections of the nuclide $j$ forming nuclide $i$. The diagonal elements, $c_{i i}=\sigma_{i}^{t}$, are the negative of the total destruction microscopic cross-section of the nuclide $i$,

$D=$ the decay matrix whose diagonal elements, $d_{i i}$, are the negative of the decay constant of the nuclides, and the off-diagonal elements, $d_{i j}$ is the partial decay constant of the $j^{\text {th }}$ nuclide decaying to the $i^{\text {th }}$ nuclide,

$A=\phi C+D$ during operation, and $A=D$ during shutdown.

The elements of $C$ and $D$ are constants. The neutron flux may, however, change with time. In fission reactors the radioactive inventory is primarily due to the production of the fission products and thus depends strongly on the fuel burn-up that in turn changes the neutron flux. In fusion reactors, the neutron flux is due to the 
external plasma neutron source. As the materials interact with neutrons, the isotopes inventory changes. Such change could, in theory, alter the neutron spectrum. However, this change in the neutron spectrum, for all practical purposes, is small and can be neglected. As a result, the coefficients in Eq. 1 are considered constants.

The $i^{\text {th }}$ row in Eq. 1 represents the rate of change of the $i^{\text {th }}$ nuclide due to its destruction and the transmutations of the other nuclides to it. The off-diagonal elements of the $j^{\text {th }}$ column in the matrix $A$ are the different transmutations of the $j^{t h}$ nuclide to the other nuclides. Since $a_{j j}$ is the negative of the total destruction cross-section, the summation of each column must equal zero, i.e. $\sum_{i} a_{i j}=0$. Apart from the fission reaction, this condition implies the conservation of the total number of nuclides in the transmutation reactions. Accordingly, the sum of the concentration vector $y$ at any time must equal the sum of the initial concentration vector, i.e. $\sum y_{i}(t)=\sum y_{i}\left(t_{0}\right)$

The decay matrix $D$ is a triangular matrix, but the cross-section matrix $C$ is, in general, not triangular. This is due to reactions such as $(n, 2 n)$ that lead to feedback loops. Therefore, the diagonal elements of $A$ are not generally identical to the eigenvalues of $A$.

The solution of Eq. 1 is given by[3]:

$$
y(t)=\exp \left(\left(t-t_{0}\right) A\right) y\left(t_{0}\right)=R\left(t, t_{0}\right) y\left(t_{0}\right)
$$

where $y\left(t_{0}\right)$ is the initial concentration vector, and matrix $R \equiv \exp (t A)$ is called the resolvent or the transition matrix. The function $\exp (t A)$ can be formally defined by the convergent power series:

$$
\exp (t A)=I+t A+\frac{t^{2} A^{2}}{2 !}+\cdots=\sum_{i=0}^{\infty} \frac{t^{i} A^{i}}{i !},
$$

where $I$ is the identity matrix. 


\section{Methods of Solutions}

The solution of Eq. 1 can be found with many different methods. For a review of many of these methods, the reader is referred to the article by Moler and Van Loan[4]. In this section, we first review briefly historical and popular methods that have been used in the transmutation calculations. Then, in the next sections, we describe the matrix decomposition methods, the Padé approximation method, and a special method for lower triangular transmutation matrices.

\subsection{Linear Chain Method}

When Rutherford[8] established the possibility of the spontaneous transmutation of elements about a century ago, he deduced mathematically the solutions of the densities of the atoms involved in a successive radioactive-decay series. Rutherford considered limited cases in which there are only two products in addition to the primary substance. Bateman[9] introduced a more generalized mathematical method that can treat unlimited number of consecutive products.

The applicability of Bateman's method is confined to naturally occurring or artificially produced radioactive substances with linear successive transmutations (A.B.C...). The linearity, in this context, means a unique path from nuclide to nuclide with no feedbacks from daughters to parents. The linearity breaks down when the nuclides in the chain are subjected to nuclear reactions e.g. neutron reactions. In this case, different paths can lead to the same isotope with possible feedback (loops) from the products to their parents (e.g. $A(n, \gamma) B(n, 2 n) A$; or $A(n, p) B\left(\beta^{-}\right) A$ ) or grandparents (e.g. $\left.A(n, \gamma) B(n, d) C\left(\beta^{-}\right) A\right)$. The system of differential equations then becomes highly coupled. This is indeed the case in fission and particularly in fusion reactors, where the high energy neutrons are capable of producing many loops.

In order to use the linear chain method to calculate the transmutations in a nuclear reactor, approximations have to be made. One may either neglect the loops altogether[10], or deal with only isolated first order loops[11], or ignore many nuclides and introduce fictitious chains and fictitious nuclides to decouple the differential equations[12]. These approximations can be justified for particular problems or chains, but can not be generalized. 


\subsection{Ordinary Differential Equation Methods}

Another approach for calculating the nuclear transmutation is the direct numerical integration of the radioactivity differential equations using an ordinary differential equation solver. The transmutation system of equations is an extremely stiff system with time constants that vary from fractions of a second to thousands of years. This requires the use of specialized methods such as the backward differentiation formulas (BDF) used in the well-tested GEAR package[13] which has automatic step size control.

Such methods, however, are designed for general nonlinear differential equations and thus do not take advantage of the linearity and the constant coefficient nature of Eq. 1. Nonetheless, these methods provide more accurate accounting of the transmutations than the linear chain method. The radioactivity code RACC[5] uses the GEAR method. Another version of RACC[6] makes use of the LSODES solver[7] which is based also on the BDF method but designed for sparse systems.

\subsection{Series Methods}

The power series definition of $\exp (t A), \mathrm{Eq} .3$, is the basis of these methods. Direct calculation of the series expansion is inefficient and unsatisfactory. However, using the identity

$$
e^{A}=\left(e^{A / m}\right)^{m},
$$

can improve the efficiency and the accuracy of these methods[14]. This is done by scaling the matrix by $m$, which is taken as a power of two, $m=2^{k}$, such that $\|A\| / m \leq 1$. The series is calculated for the scaled matrix and the result is then squared repeatedly. This scaling and squaring technique has been used in the ORIGEN code[15] to calculate Eq. 3.

\section{Matrix Decomposition Methods}

The matrix decomposition methods are based on the transformation of a matrix to a form that can be easily used. Generally this takes the form:

$$
A=S B S^{-1} \text {. }
$$


Since $S S^{-1}=I$, any power $i$ of $A$ can be written as

$$
A^{i}=S B^{i} S^{-1}
$$

Using this in the above power series definition of $e^{t A}$, Eq. 3, we find:

$$
e^{t A}=S e^{t B} S^{-1}
$$

The closer $B$ is to diagonal form, with invertible and well-conditioned $S$, the easier is the evaluation of $e^{t B}$.

\subsection{Eigenvector Decomposition}

If $A$ is an $n \times n$ matrix that has $n$ independent eigenvectors $\left[v_{1}|\cdots| v_{n}\right]=V$ with corresponding eigenvalues $\lambda_{1, \ldots,}, \lambda_{n}$, then $S$ and $B$ can be set to $V$, and $\Lambda$, respectively. Where $\Lambda=\operatorname{diag}\left(\lambda_{1}, \cdots, \lambda_{n}\right)$. Then:

$$
e^{t A}=V \operatorname{diag}\left(e^{t \lambda_{1}}, \cdots, e^{t \lambda n}\right) V^{-1}
$$

The difficulties in this approach are that $A$ does not always have a full set of independent eigenvectors and $V$, even if it is invertible, can behave very badly[16]. Another problem that has been encountered is that $A$ can sometimes have complex eigenvalues thus forcing the costly use of complex arithmetic in Eq. 8 .

\subsection{Schür Decomposition}

For any real matrix $A$, there exists an orthogonal matrix $Q$ (the Schür vectors of $A$ ), and a block upper triangular matrix $T$ (the Schür form of $A$ )such that:

$$
Q^{T} A Q=\left[\begin{array}{cccc}
T_{11} & T_{12} & \cdots & T_{1 n} \\
0 & T_{22} & \cdots & T_{2 n} \\
\vdots & \vdots & \ddots & \vdots \\
0 & 0 & \cdots & T_{n n}
\end{array}\right]
$$


where each $T_{i i}$ is either a 1-by-1 block matrix or 2-by-2 matrix having complex conjugate eigenvalues. This means that $A=Q T Q^{T}$, and substituting this equation in Eq. 3, we get

$$
e^{t A}=Q e^{t T} Q^{T}
$$

This requires the evaluation of the exponential of a triangular matrix.

Using the properties of triangular matrices, Parlett[17] showed that for any analytic function $\Phi$ of a block upper triangular matrix $T, \Phi(T) \equiv F$, is a also block upper triangular matrix with the same block structure as $T$ and commutes with $T$, i.e.

$$
F T=T F .
$$

The diagonal blocks of $F$ can be obtained from $F_{i i}=\Phi\left(T_{i i}\right)$ and the upper diagonal blocks $i<j$ may be computed one diagonal at a time, moving out from the main diagonal, from the recursive relation:

$$
T_{i i} F_{i j}-F_{i j} T_{j j}=\sum_{k=0}^{j-i+1}\left(F_{i, i+k} T_{i+k, j}-T_{i, j-k} F_{j-k, j}\right), \quad i<j .
$$

If the transmutation matrix $A$ has only real eigenvalues, $T$ and $F$ will have only $1 \times 1$ blocks, i.e. simple triangular matrices, and the computation of $F$ is straightforward. If $A$ possesses some complex eigenvalues, the $F_{i j}$ blocks may be $1 \times 1,2 \times 1$, $1 \times 2$, or $2 \times 2$, depending on the structure of $T$. The $2 \times 2$ case requires the solution of four linear algebraic equations. The case of confluent eigenvalues requires special treatment $[18]$ that uses the derivatives of the function $\Phi$.

\subsection{Matrix Decomposition Algorithm}

The algorithm uses different routines from the mathematical library LAPACK[20]. The transmutation matrix is balanced and reduced to the Hessenberg matrix $H$, which is zero below the first subdiagonal, giving $A=G H G^{T}$, where $G$ is orthogonal. The Hessenberg matrix is then reduced to the Schür form $T$ as $H=S T S^{T}$, where $S$ is the Schür vectors of $H$. Thus, $A=G S T(G S)^{T}$ and $G S$ equals $Q$ in Eq. 9. 
At this point, all the eigenvalues of $A$ are known (the diagonal of $T$ ). If required and if the eigenvalues are all real, the eigenvectors, may be computed and Eq. 8 is used to compute the resolvent. Otherwise, Eqs. 12 and 10 are used. In either case, most of the computation time is spent in the decomposition stage and is independent of the time $t$. Only at the last stage, the time $t$ is used to compute $e^{t T}$ (or $e^{t \Lambda}$ ). This makes these methods more valuable and amenable to the treatments of pulsed operation in terms of computational time saving as is discussed shortly.

\subsection{The Padé Approximation}

In this work, the Padé approximation of the matrix exponential may be used to compute the exponential of the $2 \times 2$ diagonal blocks in Eq. 9 , or may be used to calculate the resolvent $e^{t A}$ directly. In both cases, the method proved to be efficient and reliable. The $p^{t h}$ diagonal Padé approximation of $e^{A}$ is giving by:

$$
N_{p}^{-1}(-A) N_{p}(A)
$$

where

$$
N_{p}(A)=\sum_{k=0}^{p} c_{k} A^{k}, \quad c_{k}=\frac{(2 p-k) ! p !}{(2 p) ! k !(p-k) !}
$$

Ward[21] developed an algorithm that balances the matrix and makes use of the scaling and squaring technique (Eq. 4) to compute the exponential of an arbitrary matrix using the above equation. In addition, the algorithm provides an estimate of the accuracy of the results. We have used Ward's algorithm and a modified version written by Van Loan[22] that provides more control on the degree of the approximation $(p)$ and the accuracy.

\section{Triangular Systems}

Naturally, if the transmutation matrix has a special shape, one can exploit such shape in order to reduce the computation time. As described above, during shutdown and in the linear chain method, the transmutation matrices are triangular having their eigenvalues on the diagonal. One can then make use of Eq. 12 directly to evaluate the resolvents. In fact, this equation is an elegant form of the Bateman equations that 
allows for the simultaneous solution of the system of equations, even with multiple paths to the same nuclide, without the need of the artificial break of the system as in the linear chain method.

Rewriting Eq. 12 for a lower triangular matrix, we have:

$$
f_{i j}=\frac{1}{t_{i i}-t_{j j}}\left[t_{i j}\left(f_{i i}-f_{j j}\right)+\sum_{k=j+1}^{i-1}\left(f_{i k} t_{k j}-t_{i k} f_{k j}\right)\right], \quad i>j .
$$

Consider for example the following transmutation matrix and the corresponding resolvent for the following linear chain

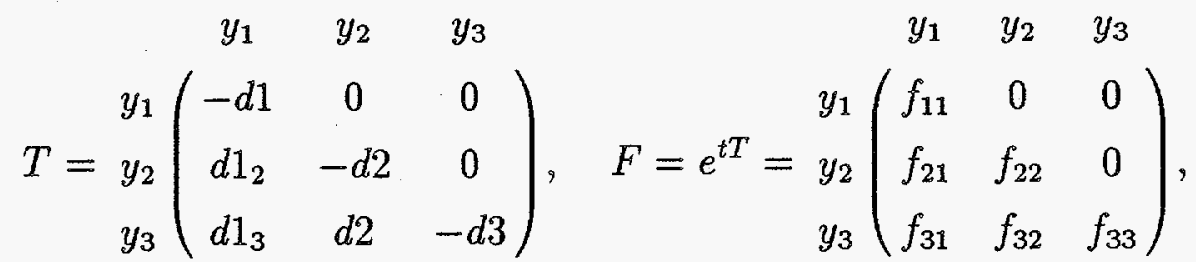

truncated for only three isotopes. The diagonal elements $f_{i i}$ are $f_{11}=e^{-t d 1}, f_{22}=$ $e^{-t d 2}$, and $f_{33}=e^{-t d 3}$. The off-diagonal elements can then be found recursively, one subdiagonal after another, using Eq.13 as:

$$
\begin{aligned}
f_{21} & =\frac{t_{21}}{t_{22}-t_{11}}\left(f_{22}-f_{11}\right)=\frac{d 1_{2}}{d 1-d 2}\left(e^{-t d 2}-e^{-t d 1}\right), \\
f_{32} & =\frac{t_{32}}{t_{33}-t_{22}}\left(f_{33}-f_{22}\right)=\frac{d 2}{d 2-d 3}\left(e^{-t d 3}-e^{-t d 2}\right), \\
f_{31} & =\frac{1}{t_{33}-t_{11}}\left[t_{31}\left(f_{33}-f_{11}\right)+f_{32} t_{21}-t_{32} f_{21}\right] \\
& =\frac{1}{d 1-d 3}\left[d 1_{3}\left(e^{-t d 3}-e^{-t d 1}\right)+\frac{d 1_{2} d 2}{d 2-d 3}\left(e^{-t d 3}-e^{-t d 2}\right)-\frac{d 2 d 1_{2}}{d 1-d 2}\left(e^{-t d 2}-e^{-t d 1}\right)\right] .
\end{aligned}
$$

The same procedure can be used to evaluate the power of the triangular matrix, say $R^{m}$, with the diagonal elements $f_{i i}=r_{i i}^{m}$. 


\section{Pulsed Operation}

We previously examined analytically the effect of pulsed operation in ITER and showed how it depends on the burn time, the dwell time, and the half-lives[1]. That analysis showed also that for ITER's low duty factor, using the continuous operation assumption would considerably overestimate the radioactivities, for a wide range of half-lives. For a few number of pulses, the nuclear transmutations can be computed by any of the methods described above by solving the transmutation equations for each pulse and shutdown periods. For a large number of periodic sequences of pulses, one can save a significant amount of computing time by taking advantage of the regularity in the pulse sequence. This can be done if the solutions are obtained in terms of the resolvents of the different periods. As seen in Eq. 2, the resolvent is constant for the same transmutation matrix and the same time difference.

For a uniform sequence of pulses, suppose that $R_{p}\left(t_{p}\right)$ is the resolvent during the pulse time $t_{p}$, and $R_{d}\left(t_{d}\right)$ is the resolvent during the decay time $t_{d}$ between pulses. Using Eq. 2 the solution at the end of $t_{d}$ is

$$
y\left(t_{d}+t_{p}\right)=R_{d} R_{p} y(0) \equiv R_{c} y(0),
$$

where $R_{c}$ is the resolvent for one cycle of a pulse and a decay period. If this cycle is repeated $m$ times, the solution at the end of the $m^{\text {th }}$ pulse is

$$
y\left(m t_{p}+(m-1) t_{d}\right)=R_{p} R_{c}^{m-1} y(0)
$$

This requires at most $2\left[\log _{2}(m)\right]$ matrix multiplications[23].

Depending on the size of the matrix $n$ and the number of pulses $m$, computing cost could be reduced by decomposing $R_{c}$ itself using the matrix decomposition methods discussed above. Using the eigenvector decomposition

$$
R_{c}^{m-1}=\operatorname{Vdiag}\left(\lambda_{1}^{m-1} \ldots \lambda_{n}^{m-1}\right) V^{-1},
$$


where $V$ and $\lambda_{1} \ldots \lambda_{n}$ are the eigenvectors and the eigenvalues of $R_{c}$, and using the Schür decomposition

$$
R_{c}^{m-1}=Q T^{m-1} Q^{T}
$$

where $Q$ and $T$ are the Schür vectors and form of $R_{c}$. The same matrix decomposition algorithm described above is used with the function $\Phi(x)=x^{m}$.

For triangular transmutation systems, $R_{p}$ and $R_{d}$, are triangular and Eq. 13 can then be used to evaluate the powers of the resolvent of the cycle $R_{c}$, which is also triangular. Thus, the decomposition stage including the calculation of the eigenvalues of the triangular matrices[24] is totally unnecessary.

\section{An Example Problem}

The above algorithms described above have been coded for the CRAY computers which have 64-bit word and 47-bit mantissa. We demonstrate the results of the different methods for a problem used to compare different transmutation codes[25] utilizing reduced cross-section and decay data libraries and a specified neutron flux. The problem is the evaluation of the transmutation of ${ }^{50} \mathrm{Cr}$ after one year of irradiation. The initial atomic concentration of ${ }^{50} \mathrm{Cr}$ is set to 10 . The transmutation matrix, with the reaction modes, is shown in Fig. 1.

Notice that the submatrix of the $\mathrm{Cr}$ isotopes is triangular and the analytical solutions of these isotopes can be found using Eq. 13. Table 1 shows this analytical solution, the results of the different methods, the deviations from the initial concentration giving in particles per million, and the CPU times in milli seconds. The results of the $\mathrm{Cr}$ isotopes from the different methods are shown as the percentage differences from the analytical solutions. For the other isotopes, the results are given in absolute values.

For the $\mathrm{Cr}$ isotopes, the solutions of the matrix decomposition methods are the closest to the analytical solutions. The differences from the analytical solutions of these methods are orders of magnitude less than the other methods. The Schür method is the most accurate method. There is considerable reduction in the CPU 
章

蔡

$\overrightarrow{7}$

客

密

$\sum_{\frac{1}{2}}^{\infty} \ldots \ldots \ldots \ldots$

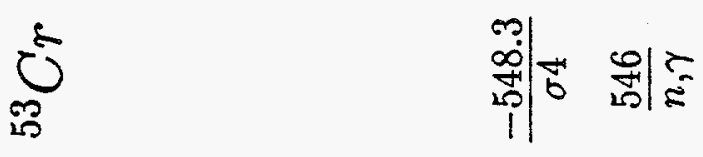

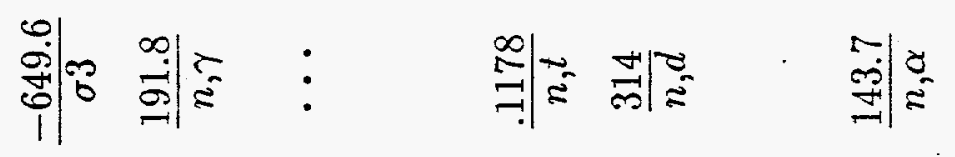

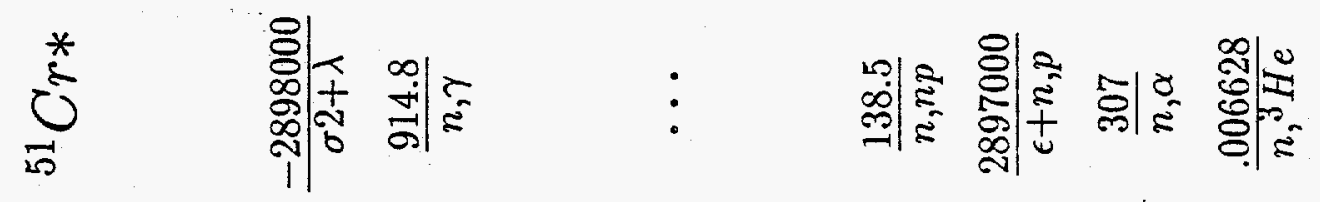

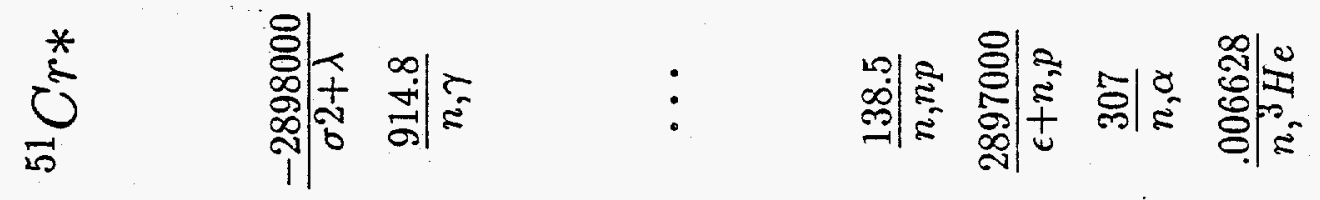

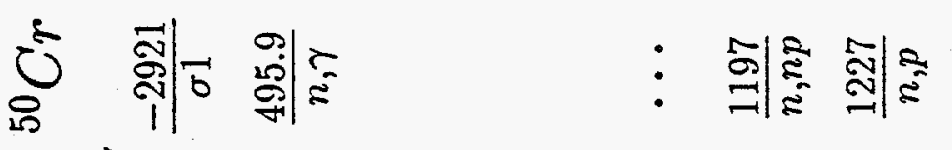

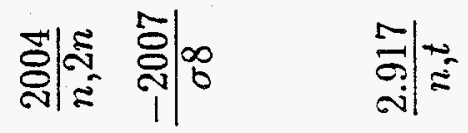

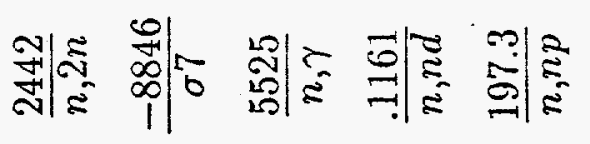

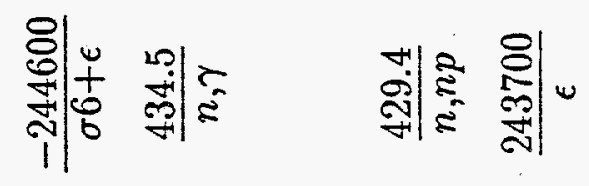

예예

iิ

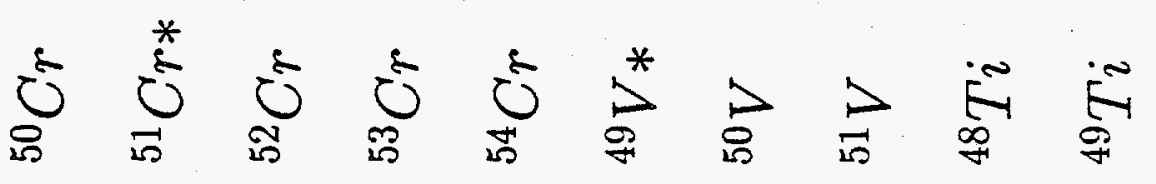

竞 
times by using the decomposition methods and the Padé method. The LSODES method has the largest CPU time. However, this could be balanced, to some extent, by the fact that this method requires the minimum memory. It should be noted that the CPU time depends on the size of the matrix and the saving in computing time could be greater for larger problems by using the decomposition methods.

Consider now the same problem for pulsed operation. To reach the same fluence used in the continuous irradiation calculations above, we assume 10000 pulses with a pulse width and a dwell time of 3156 and 2000 seconds, respectively. Table 2 shows the results, using the eigenvector decomposition, at the end of the first pulse, at the end of the first decay period, and at the end of the 10000th pulse. The computing time was only $31 \mathrm{~ms}$. This is remarkably orders of magnitude less than that for the backward differentiation formulas (BDF) methods which can be estimated from Table $^{\sim} 1$ as at least $10000 \times 2 \times 13=260000 \mathrm{~ms}$.

\section{Application to ITER}

We apply now the above pulsed algorithm to a recent ITER design and for different operation scenarios. This design is for the shielding blanket (SS/water). The radial build of this design is shown in Fig. 2. The neutron flux was calculated using the neutron transport code ONEDANT[26]. Figure 2 shows also the neutron flux grouped in fewer groups, for illustrative purpose, than the 46-neutron and the 21-photon groups used in the transport and the activation calculations.

The neutron wall loading on the inboard first wall is about $.92 \mathrm{MW} / \mathrm{m}^{2}$. Three operational scenarios are assumed to reach the target design fluence of $3 \mathrm{MWa} / \mathrm{m}^{2}$. The first is simply the continuous operation mode, for 3.25 years of operation. Assuming a burn time of 1000 seconds and a dwell time of 2039 seconds, the second scenario assumes 103652 cycles. The third scenario assumes 101777 cycles followed by a two weeks of continuous operation.

The specific activity, the decay heat, and the contact dose in the inboard first wall, for the three operation scenarios, are shown in Fig 3-5. It is clear that the continuous operation mode overestimates the specific activity, the decay heat, and 
Table 1: Solution Comparison- Transmutation of ${ }^{50} \mathrm{Cr}$ after one year of continuous operation $^{a}$

\begin{tabular}{|c|c|l|l|}
\hline Isotope & \multicolumn{1}{|c|}{ Analytical } & \multicolumn{1}{|c|}{ Schür } & \multicolumn{1}{|c|}{ Eigenvector } \\
\hline${ }^{50} \mathrm{Cr}$ & 9.90824823398 & $0.0 \%$ & $0.0 \%$ \\
${ }^{51} \mathrm{Cr} *$ & $1.69666077856 \mathrm{E}-03$ & $-1.77085549354 \mathrm{E}-10 \%$ & $-1.77085549354 \mathrm{E}-10 \%$ \\
${ }^{52} \mathrm{Cr}$ & $4.37693012077 \mathrm{E}-06$ & $-1.13945844595 \mathrm{E}-10 \%$ & $-1.13945844595 \mathrm{E}-10 \%$ \\
${ }^{53} \mathrm{Cr}$ & $1.19872826563 \mathrm{E}-09$ & $4.17120502630 \mathrm{E}-09 \%$ & $-2.26072577552 \mathrm{E}-07 \%$ \\
${ }^{54} \mathrm{Cr}$ & $6.29810678824 \mathrm{E}-13$ & $-7.19772138009 \mathrm{E}-07 \%$ & $8.83054064462 \mathrm{E}-04 \%$ \\
\hline${ }^{49} \mathrm{~V}^{*}$ & NA & $2.63103404748 \mathrm{E}-02$ & $2.63103404748 \mathrm{E}-02$ \\
${ }^{50} \mathrm{~V}$ & NA & $3.80864589509 \mathrm{E}-02$ & $3.80864589509 \mathrm{E}-02$ \\
${ }^{51} \mathrm{~V}$ & NA & $1.41676438411 \mathrm{E}-02$ & $1.41676438410 \mathrm{E}-02$ \\
${ }^{48} \mathrm{Ti}$ & NA & $5.54928385814 \mathrm{E}-05$ & $5.54928383281 \mathrm{E}-05$ \\
${ }^{49} \mathrm{Ti}$ & NA & $1.13900571692 \mathrm{E}-02$ & $1.13900571714 \mathrm{E}-02$ \\
Error-ppm & 0.0 & -4.07338371247 & -4.07338350783 \\
$\mathrm{CPU}(\mathrm{ms})$ & & 4 & 5 \\
\hline \hline Isotope & Padé & GEAR & LSODES \\
\hline${ }^{50} \mathrm{Cr}$ & $0.0 \%$ & $0.0 \%$ & $0.0 \%$ \\
${ }^{51} \mathrm{Cr} *$ & $6.30849143866 \mathrm{E}-05 \%$ & $-5.19315949417 \mathrm{E}-05 \%$ & $-4.94088313428 \mathrm{E}-07 \%$ \\
${ }^{52} \mathrm{Cr}$ & $-7.71967986919 \mathrm{E}-06 \%$ & $6.35502480098 \mathrm{E}-06 \%$ & $5.99739090522 \mathrm{E}-08 \%$ \\
${ }^{53} \mathrm{Cr}$ & $1.01357411537 \mathrm{E}-06 \%$ & $-2.39086670990 \mathrm{E}-06 \%$ & $-7.43287647805 \mathrm{E}-07 \%$ \\
${ }^{54} \mathrm{Cr}$ & $1.59468124902 \mathrm{E}-03 \%$ & $1.59707117337 \mathrm{E}-03 \%$ & $1.59571711576 \mathrm{E}-03 \%$ \\
\hline${ }^{49} \mathrm{~V}^{*}$ & $2.63103404748 \mathrm{E}-02$ & $2.63103404719 \mathrm{E}-02$ & $2.63103404678 \mathrm{E}-02$ \\
${ }^{50} \mathrm{~V}$ & $3.80864589516 \mathrm{E}-02$ & $3.80864589504 \mathrm{E}-02$ & $3.80864589502 \mathrm{E}-02$ \\
${ }^{51} \mathrm{~V}$ & $1.41676427705 \mathrm{E}-02$ & $1.41676447224 \mathrm{E}-02$ & $1.41676438497 \mathrm{E}-02$ \\
${ }^{48} \mathrm{Ti}$ & $5.54928394367 \mathrm{E}-05$ & $5.54928396125 \mathrm{E}-05$ & $5.54928396451 \mathrm{E}-05$ \\
${ }^{49} \mathrm{Ti}$ & $1.13900571682 \mathrm{E}-02$ & $1.13900571712 \mathrm{E}-02$ & $1.13900571751 \mathrm{E}-02$ \\
Error-ppm & -4.07338370678 & -4.07338372383 & -4.07338367268 \\
$\mathrm{CPU}(\mathrm{ms})$ & 3 & 13 & 55 \\
\hline
\end{tabular}

(a) Values in $\%$ are $\left(y_{\text {meth }}-y_{\text {anal }}\right) / y_{\text {anal }} * 100$.

(b) Error $=10^{6}\left(\sum y_{i}(t)-\sum y_{i}(0)\right) / \sum y_{i}(0)$ 


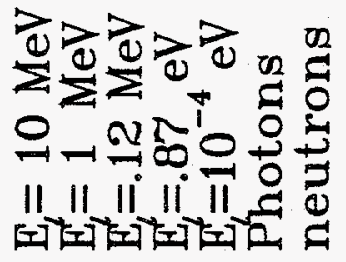
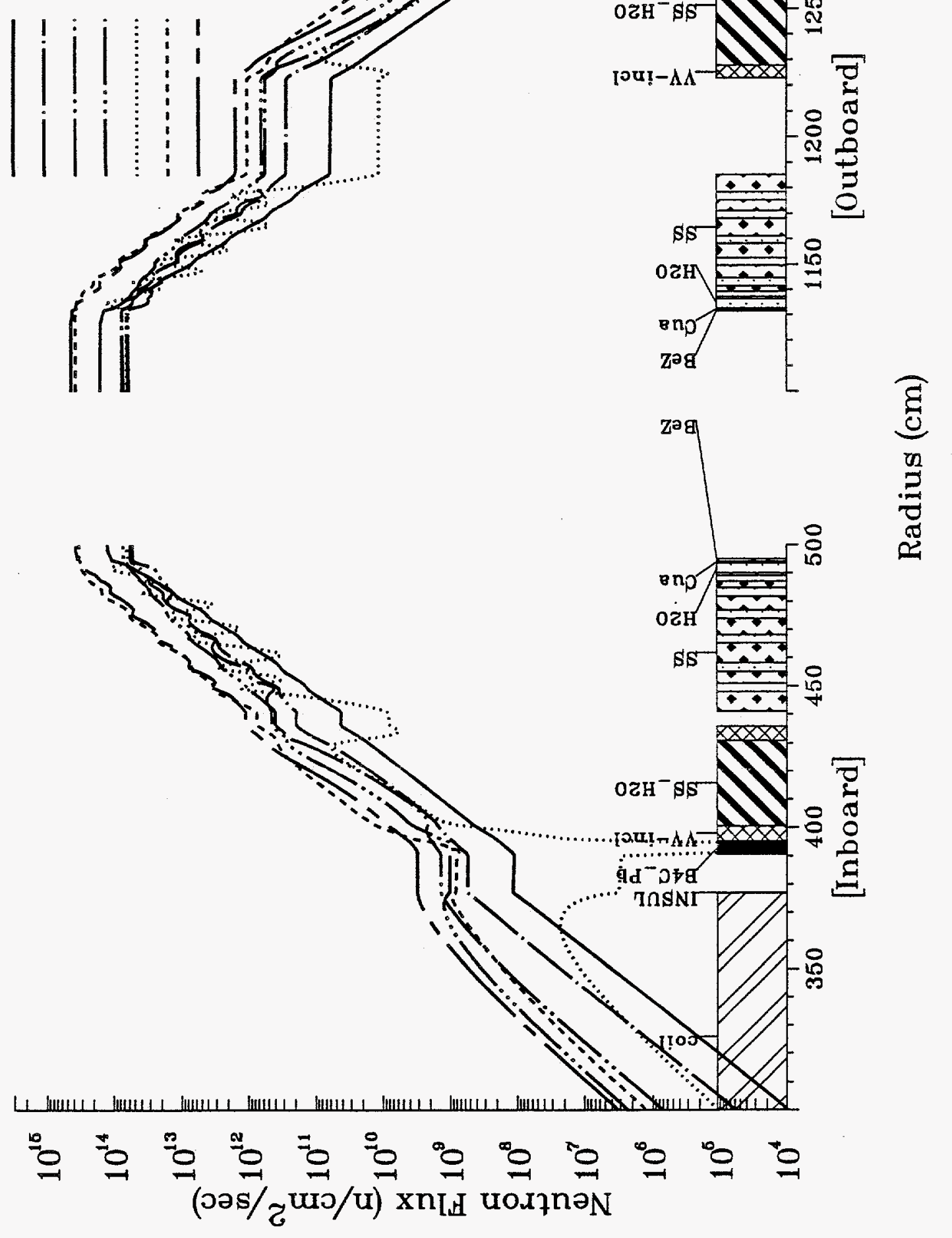

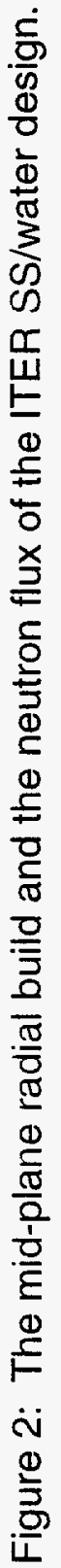


Table 2: Transmutation of ${ }^{50} \mathrm{Cr}$ after 10000 pulses ${ }^{a}$.

\begin{tabular}{|c|l|l|l|}
\hline Isotope & \multicolumn{1}{|c|}{ End Pulse } & \multicolumn{1}{|c|}{ End Dwell } & 10000 Pulse \\
\hline${ }^{50} \mathrm{Cr}$ & 9.999991 & 9.999991 & 9.908248 \\
${ }^{51} \mathrm{Cr}^{*}$ & $1.564205 \mathrm{E}-06$ & $1.563300 \mathrm{E}-06$ & $1.038884 \mathrm{E}-03$ \\
${ }^{52} \mathrm{Cr}$ & $2.258176 \mathrm{E}-13$ & $2.258176 \mathrm{E}-13$ & $2.806988 \mathrm{E}-06$ \\
${ }^{53} \mathrm{Cr}$ & $2.032879 \mathrm{E}-17$ & $2.032879 \mathrm{E}-17$ & $7.977416 \mathrm{E}-10$ \\
${ }^{54} \mathrm{Cr}$ & 0.0 & 0.0 & $1.819256 \mathrm{E}-13$ \\
${ }^{49} \mathrm{~V}^{*}$ & $3.778731 \mathrm{E}-06$ & $3.778547 \mathrm{E}-06$ & $2.148223 \mathrm{E}-02$ \\
${ }^{50} \mathrm{~V}$ & $3.873765 \mathrm{E}-06$ & $3.873765 \mathrm{E}-06$ & $3.808704 \mathrm{E}-02$ \\
${ }^{51} \mathrm{~V}$ & $7.185008 \mathrm{E}-10$ & $1.624175 \mathrm{E}-09$ & $1.482433 \mathrm{E}-02$ \\
${ }^{48} \mathrm{Ti}$ & 0.0 & 0.0 & $6.830789 \mathrm{E}-05$ \\
${ }^{49} \mathrm{Ti}$ & $1.477929 \mathrm{E}-10$ & $3.315157 \mathrm{E}-10$ & $1.620743 \mathrm{E}-02$ \\
Error-ppm & $4.445155 \mathrm{E}-6$ & $4.439471 \mathrm{E}-6$ & -4.072662 \\
$\mathrm{CPU}(\mathrm{ms})$ & & & 3 \\
\hline
\end{tabular}

(a) Burn time $=3156$ seconds and dwell time $=2000$ seconds.

(b) Error $=10^{6}\left(\sum y_{i}(t)-\sum y_{i}(0)\right) / \sum y_{i}(0)$.

the contact dose, at the final shutdown and for about one week after the shutdown, by about a factor of 2.4 more than the pure pulsed operation mode. Adding a short period of continuous operation (two weeks as in the third scenario) decreases this factor considerably. The differences between the different operation scenarios decreases afterward and vanishes, as expected[1], after a long cooling time.

One expects that for an experimental device such as ITER, there would be longer shut-down periods between pulses and limited numbers of long burn-time pulses. This would make the radioactivity inventory even less than that of the pure pulsed scenario. Thus, it is important in order to alleviate safety constraints on ITER design, to have a reasonable estimate of the operation scenario of ITER.

\section{Summary and Conclusions}

All the objectives of this design task have been met. We have demonstrated the use of different mathematical methods for solving the transmutation system of equations in steady-state and pulsed operation fusion reactors. Considerable accu- 
racy and efficiency can be achieved by using the matrix decomposition methods. A simple recursive relation has been developed to calculate the resolvents in triangular transmutation systems ( linear chain methods). The results for a simple problem show the accuracy and the efficiency of the different methods, and the saving in computing times by using the matrix decomposition methods in pulsed operation. The calculations for a recent ITER design show the large difference between the continuous operation assumption, which is usually used in the activation calculations, and a conservative pulsed operation scenarios. 


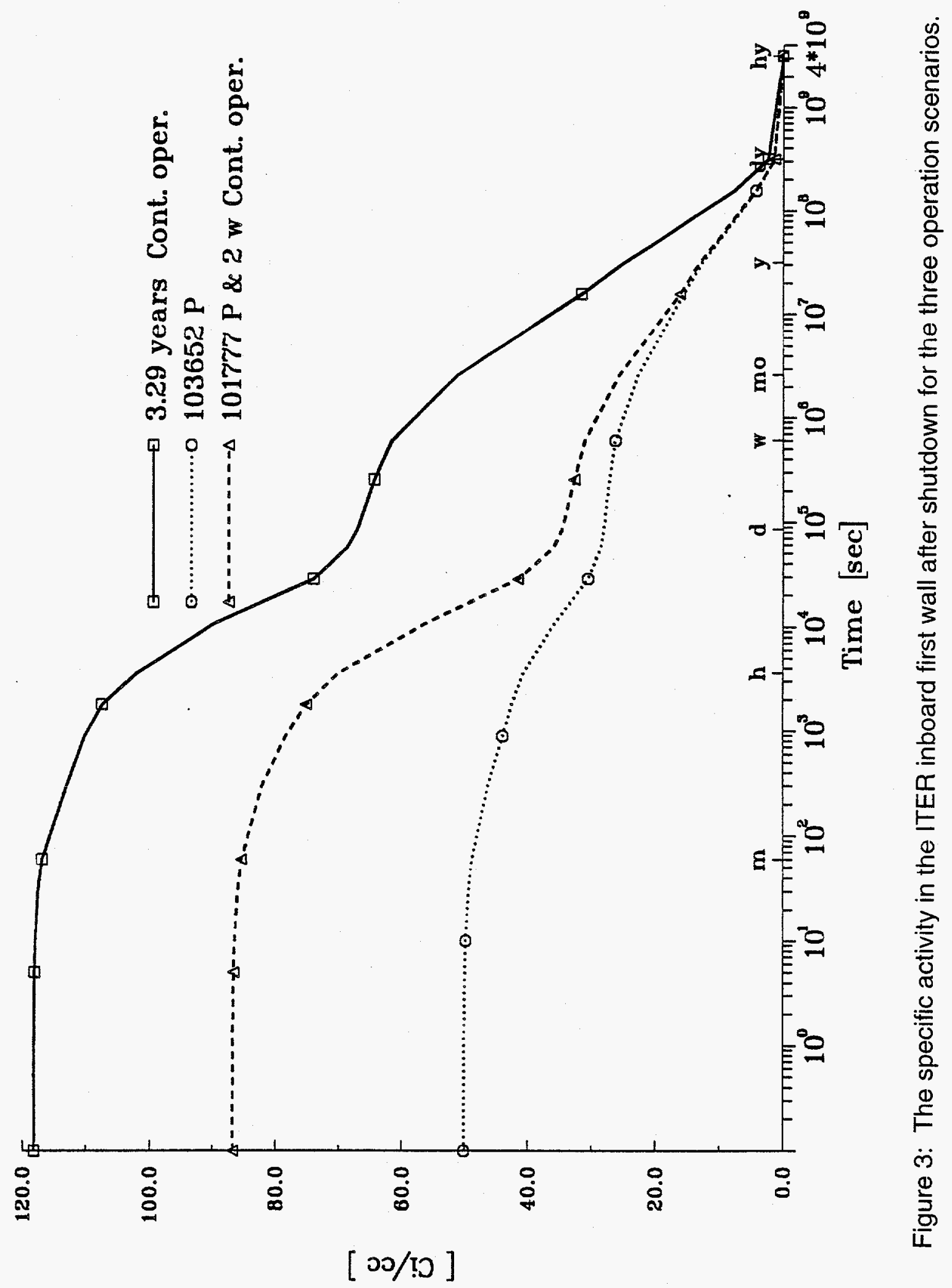




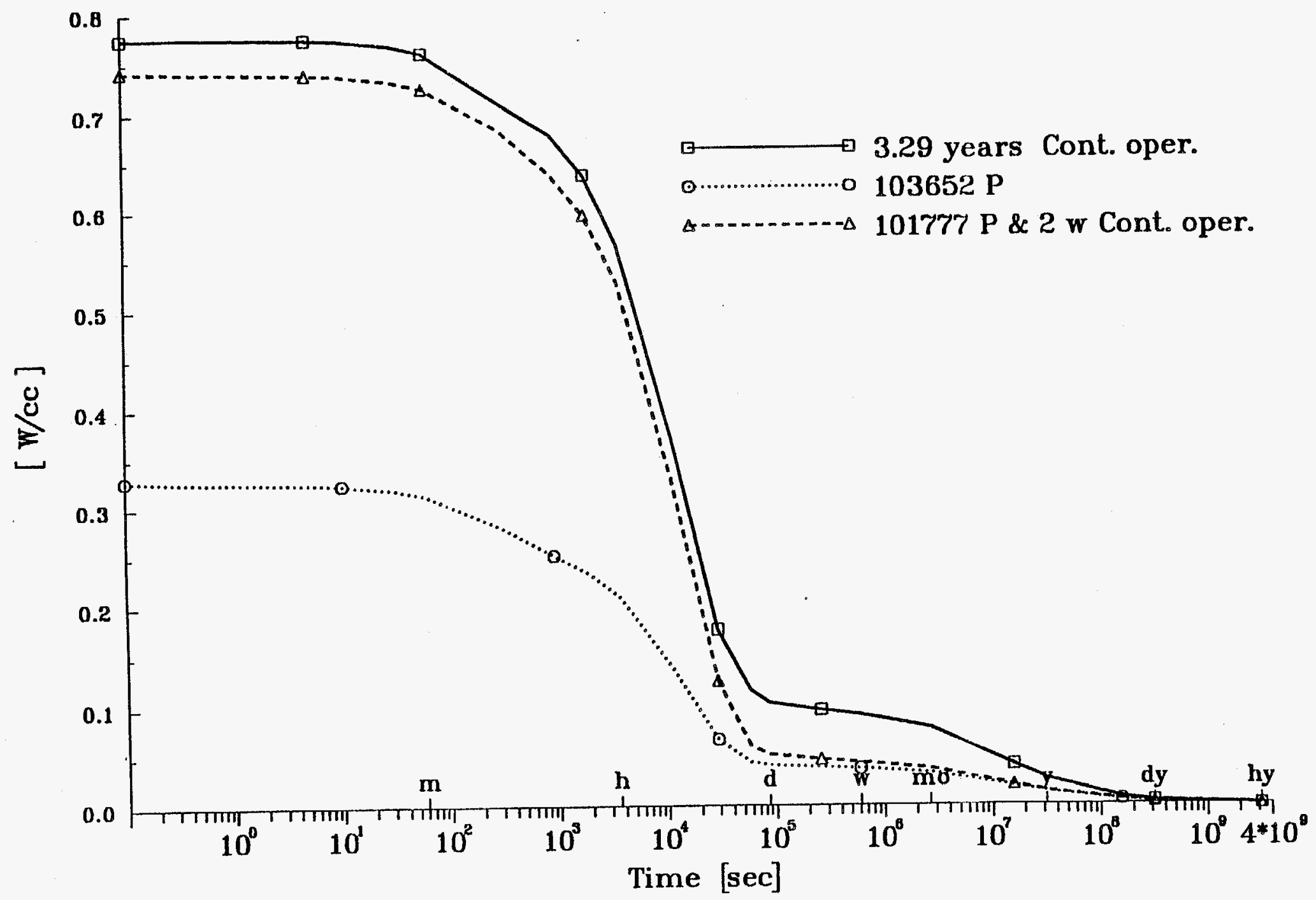

Figure 4: The specific decay heat in the ITER inboard first wall after shutdown for the three operation scenarios. 


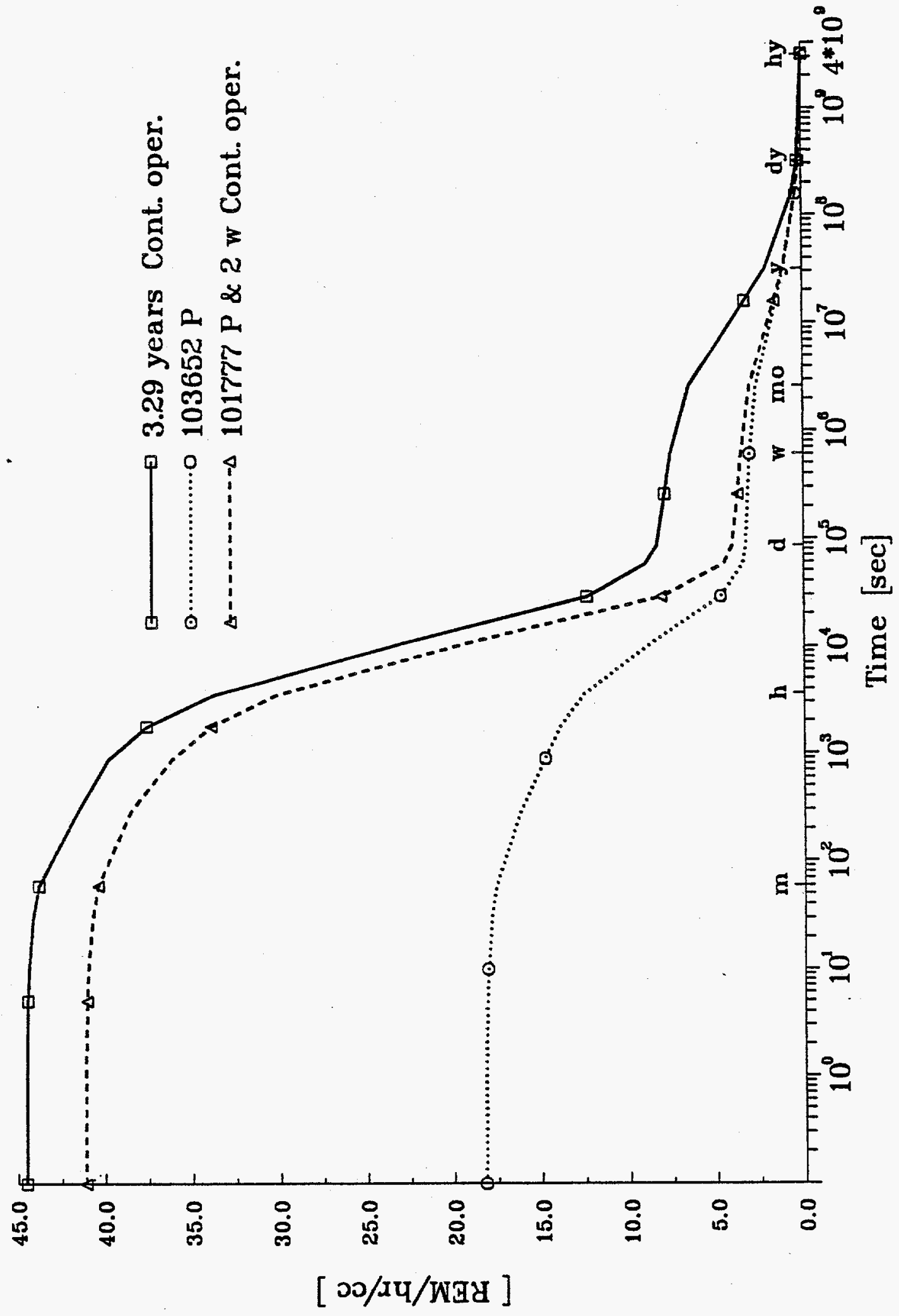

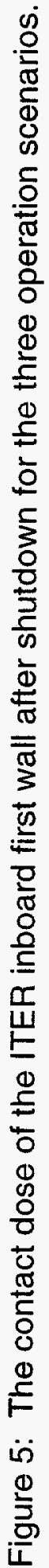




\section{References}

[1] H. Attaya, Y. Gohar, and D. Smith. US-ITER activation analysis. Fusion Technology 19(1991)1837.

[2] H. Attaya. Improvement of decay and cross sections data libraries for activation calculation. DOE/ER-0313/14 ( July 1993).

[3] R. Bellman. Introduction to matrix analysis. ( $2^{\text {nd }}$ Edition, McGraw-Hill, New York, 1970) pp. 163-174.

[4] C. Moler and C. Van Loan. Nineteen dubious ways to compute the exponential of a matrix. SIAM Review 20(1978)801-36.

[5] J. Jung. Theory and use of the radioactivity code RACC. Argonne National Laboratory. ANL/FPP/TM-122 (May 1979).

[6] H. Attaya. Input instructions for RACC-P. Argonne National Laboratory. ANL/FPP/TM-270 (March 1994).

[7] A. Hindmarsh. ODEPACK, a systematized collection of ODE solvers, in: Scientific Computing, eds. R. Stepleman et al. (IMACS/North-Holland, Amsterdam, 1983) pp. 55-64.

[8] E. Rutherford. Radio-activity, (2 $2^{\text {nd }}$ Edition, Cambridge University Press, 1905.) pp. 330-337.

[9] H. Bateman. The solution of a system of differential equations occurring in the theory of radio-activity transformations. Proc. Cambridge Phil. Soc. $15(1910) 423$.

[10] T. Y. Sung and W. F. Vogelsang,. DKR: A radioactivity calculation code for fusion reactors. University of Wisconsin . UWFDM-170 (September 1976).

[11] D. Henderson and O. Yasar. DKRICF: A radioactivity and dose rate calculation code package. University of Wisconsin. UWFDM-714 (April 1987).

[12] T. England, W. Wilson, and M. Stamatelatos. Fission products data for thermal reactors, Part1: A data set for EPRI-CINDER using ENDF/B-IV. Electric Power Research Institute. EPRI NP-356 (ERDA E(29-2)-3608) (December 1976). 
[13] A. Hindmarsh. GEAR: Ordinary differential equation system solver. LLNL report, UCID-30001, Rev. 3 (December 1974).

[14] L. Lapidus and R. Luus. Optimal control of engineering process. (Blaisdell, Waltham, Mass., 1967) pp.45-49.

[15] M. Bell. ORIGEN - the ORNL isotope generation and depletion code. Oak Ridge National Laboratory. ORNL-4628 (May 1973).

[16] E. Hairer, S. Norsett, and G. Wanner. Solving ordinary differential equations I. (Springer-Veralg, Berlin 1987) p. 70.

[17] B. Parlett. A recurrence among the elements of functions of triangular matrices. Lin. Alg. and Its Applic. 14(1976)117.

[18] B. Parlett. Computation of functions of triangular matrices. Electronics Research Laboratory, University of California, Berkeley. Memorandum No. ERLM481(November 1974).

[19] J. Wilkinson. The algebraic eigenvalue problem. (Clarendon Press, Oxford, England 1965) pp

[20] E. Anderson et al. LAPACK users' guide. (SIAM, Philadelphia, 1992).

[21] R. Ward. Numerical computation of the matrix exponential with accuracy estimate. SIAM J. Numer. Anal. 14(1977)600.

[22] C. Van Loan. Computing integrals involving the matrix exponential. IEEE Trans. Auto. Cont. AC-23(1978)395.

[23] G. Golub and C. Van Loan. Matrix computations. ( $2^{\text {nd }}$ Edition, The Johns Hopkins University Press, Baltimore, 1989) p. 552

[24] S. Spangler, J. Sisolak, and D. Henderson. Calculational models for the treatment of pulsed/intermittent activation within fusion energy devices. Fusion Engineering and Design 22(1993)349-366.

[25] E. Cheng, R. Forrest, and A. Pashchenko. Report on the Second International Activation Calculation Benchmark Comparison Study. TSI Research Inc. TSIR21 (December 1993). 
[26] R.D. O'Dell et al., "User's Manual for ONEDANT: a code package for onedimensional, diffusion-accelerated, neutral particle transport," Los Alamos National Laboratory Report, LA-9184-M (1989). 


\section{DISTRIBUTION LIST FOR ANL/FPP/TM-277}

\section{Internal}

H. Attaya (10)

S. Bhattacharyya

M. Billone

J. Brooks

O. Chopra

H. Chung

D. Diercks

D. Ehst

Y. Gohar

I. Gomes

D. Gruen
A. Hassanein

H. Herman

T. Hua

A. Hull

C. Johnson

T. Kassner

J. Kopasz

A. Krauss

M. Lineberry

S. Majumdar

R. Mattas
B. Micklich

K. Natesan

J.H. Park

C. Reed

Dale L. Smith

D.-K. Sze

H. Tsai

T. Yule

FPP Files (10)

TIS Files (1)

\section{External}

DOE/OSTI for distribution per UC-424 (41)

Manager, Chicago Operations Office

ANL-E Libraries (2)

ANL-W Library

M. Abdou, University of California, Los Angeles

C. Baker, University of California, San Diego

J. Bartlit, Los Alamos National Laboratory

S. Berk, U.S. Department of Energy

E.T. Cheng, TSI Research, Inc., California

M. Cohen, U.S. Department of Energy

J. Davis, McDonnell Douglas Astronautics Company

J. Doggett, Lawrence Livermore National Laboratory

U. Fischer, Forschungszentrum Karlsruhe, Germany

D. Gelles, Pacific Northwest Laboratories

L. Greenwood, Battelle Pacific Northwest Laboratory

D. Henderson, University of Wisconsin

T. James, U.S. Department of Energy

D.C. Larson, Oak Ridge National Laboratory

S. Malang, Forschungszentrum Karlsruhe, Germany

F.M. Mann, Westinghouse Hanford Company, Washington

R. McGrath, Sandia National Laboratories, Albuquerque

R. Nygren, Sandia National Laboratories, Albuquerque

A. Opdenaker, U.S. Department of Energy

R. Parker, ITER JCT, Garching, Germany

S. Piet, ITER JCT, San Diego, California

R.W. Roussin, Oak Ridge National Laboratory

R. Price, U.S. Department of Energy

A. Raffray, ITER JCT, Garching, Germany

R.T. Santoro, ITER JCT, Garching, Germany

M. Sawan, University of Wisconsin

M. Tillack, University of California, San Diego

R. Watson, Sandia National Laboratories, Albuquerque

F.W. Wiffen, U.S. Department of Energy

K. Wilson, Sandia National Laboratories, Livermore

P.G. Young, Los Alamos National Laboratory

M. Youssef, University of California, Los Angeles

Librarian, Culham Laboratory, England 